PS 2000-060 (AE)

August 30, 2000

\title{
NEW METHODS TO DERIVE THE OPTICAL AND BEAM PARAMETERS IN TRANSPORT CHANNELS
}

\author{
G. Arduini, M. Giovannozzi, K. Hanke, D. Manglunki, M. Martini
}

\begin{abstract}
The standard approach to compute the optical parameters and the beam emittance in a transport channel is based on the analysis of the profiles measured by three monitors. This requires the independent measurement of the dispersion function at the monitor locations and the knowledge of the value of the beam's momentum spread.

In this paper different approaches based on the use of more than three beam profiles are presented. These techniques allow the determination of the complete set of four optical parameters, the betatron and dispersion functions and their derivatives, along with the beam emittance and the beam's momentum spread, simultaneously and without varying the physical parameters of the transport channel or the upstream machine.

A detailed description of the different methods is carried out with a particular emphasis on their accuracy. Results of numerical simulations are presented.
\end{abstract}

Accepted for publication in Nuclear Instr. and Methods in Physics Research A 
The complexity of the new generation of high-energy superconducting machines imposes tight constraints on the whole injector chain. In order to achieve the top performance in terms of luminosity of the planned CERN LHC [1], it is mandatory to preserve the value of the beam emittance through the whole chain of low-energy machines [2, 3]. Although a crucial point is to avoid beam blow-up in the circular accelerators, which is obtained by carefully tuning the machines, the matching of the transfer lines to the phase space of the preceding and the subsequent machine also play a predominant role.

In fact, even neglecting emittance blow-up due to the presence of possible non-linear fields, a transfer line generates transverse or longitudinal mismatch which will produce emittance increase after beam filamentation.

Hence, as a consequence of the high performance required for the physics experiments, there has been a renewed interest in optics issues in transfer lines, with special emphasis on automated algorithms to measure optical parameters with the aim of computing mismatch factors, and, eventually, of applying corrections (see Ref. [4] for an overview of this field). These algorithms are usually devoted to the correction of the beam trajectory, via dedicated steering magnets, or to reducing the injection mismatch by using quadrupole magnets. An application of these concepts to the transfer line joining the PS and the SPS machines allowed a substantial improvement of the beam quality [5].

Every correction procedure is based on a set of measured parameters to be compared with their theoretical values. Two different approaches are available. The first one deals with the measurement of the optical parameters by trajectory fit on Beam Position Monitor (BPM) data [6]. It allows the reconstruction of the transfer matrices between different BPMs and, in particular, the value of the dispersion function.

The second technique allows to derive not only the optical parameters $\alpha, \beta, \gamma$ but also the beam characteristics, emittance and beam's momentum spread, by means of a fit of beam profiles $[6,7]$. The very essence of this approach consists in looking for a 'best beam' that fits the measured data. In doing so, the optical parameters $\alpha, \beta, \gamma$, which are a characteristic of the transport channel, are mixed up with beam parameters (emittance $\epsilon$ and relative momentum spread $\delta$ ) thus introducing the possibility of spurious compensations between the two sets of parameters.

The standard is based on the measurement of the transverse beam profile at three different locations together with the measurement of the dispersion function at the same three locations $[8,9]$. The dispersion function needs to be measured independently by recording the beam position at the monitors for different values of the beam momentum and by computing the slope of a straight line fitted to the measured data. The main drawback of such an approach is that it requires modifying some machine parameters and, in some situations, the extraction process does not allow this to be done.

A possibility to avoid these off-momentum measurements, consists in exploiting the information coming from a larger number of measured beam profiles. According to the number of profiles it is possible to derive a larger set of optical $\left(\alpha, \beta, D\right.$ and its derivative $\left.D^{\prime}\right)$ and beam parameters $\epsilon, \delta$ without varying any physical parameters of the transport channel or of the preceding machine. For instance, five beam profiles allow the computation of $\alpha, \beta, D, D^{\prime}$ and $\epsilon$ [10], while the information contained in six profiles can be used to derive $\delta$ also. For more than six profiles a least squares approach can be used to improve the accuracy. These techniques are presented and discussed in detail in this paper. Analytical formulae are derived to quantify the error associated with the computed parameters and the accuracy of the different methods is tested by numerical simulations. 
In Section 2, the theory of the standard three-monitor method is reviewed and the new approaches based on five and six monitors are presented. In addition, the approach based on more than six monitors is described and a general formula to evaluate the accuracy of the different methods is introduced. In Section 3, the results of numerical simulations are reported. Finally, some conclusions are drawn in the last Section.

\section{Theory}

The standard three-monitor method [8,9] derives the optical parameters $\alpha, \beta$ and the emittance $\epsilon$ from the measurement under the following assumptions:

- The value of the dispersion function $D$ is known (possibly zero) all along the section where the monitors are installed.

- The transfer matrices of the beam line sections between the monitors are known.

- The beam dynamics in the horizontal and vertical planes is fully decoupled in between the monitors.

If $\sigma_{i}$ indicates the width of the beam profile at one sigma measured in the $i$ th monitor, then

$$
\sigma_{i}^{2}=\sigma_{\beta, i}^{2}+D_{i}^{2} \delta^{2}, \quad i=1,2,3
$$

in which $\sigma_{\beta, i}$ is the rms betatron beam width defined as

$$
\sigma_{\beta, i}=\sqrt{\beta_{i} \epsilon}
$$

and $\epsilon, \delta$ are the transverse emittance and the relative momentum spread of the beam at one sigma, respectively. Using Eq. (2) and the transformation matrix for the Twiss parameters $\left(\beta_{i}, \alpha_{i}, \gamma_{i}\right)[8]$

$$
\left(\begin{array}{c}
\beta_{i} \\
\alpha_{i} \\
\gamma_{i}
\end{array}\right)=\left(\begin{array}{ccc}
C_{i}^{2} & -2 C_{i} S_{i} & S_{i}^{2} \\
-C_{i} C_{i}^{\prime} & S_{i}^{\prime} C_{i}+S_{i} C_{i}^{\prime} & -S_{i} S_{i}^{\prime} \\
C_{i}^{\prime 2} & -2 C_{i}^{\prime} S_{i}^{\prime} & S_{i}^{\prime 2}
\end{array}\right)\left(\begin{array}{c}
\beta_{1} \\
\alpha_{1} \\
\gamma_{1}
\end{array}\right)
$$

where $C_{i}, S_{i}$ are the cosine-like and sine-like solutions for the betatron motion between the first and the $i$ th monitors, and $C_{i}^{\prime}$, $S_{i}^{\prime}$ represent the derivatives of $C_{i}$ and $S_{i}$ with respect to $s$, the betatron beam widths $\sigma_{\beta, i}$ at the three monitors may be written in terms of the emittance and the Twiss parameters at the first monitor in a $3 \times 3$ matrix formulation

$$
\Sigma=\mathcal{M} \Pi
$$

Here the three-dimensional vectors $\Sigma, \Pi$ and the matrix $\mathcal{M}$ are defined as

$$
\boldsymbol{\Sigma}=\left(\begin{array}{l}
\sigma_{\beta, 1}^{2} \\
\sigma_{\beta, 2}^{2} \\
\sigma_{\beta, 3}^{2}
\end{array}\right) \quad \boldsymbol{\Pi}=\left(\begin{array}{c}
\beta_{1} \epsilon \\
\alpha_{1} \epsilon \\
\gamma_{1} \epsilon
\end{array}\right) \quad \mathcal{M}=\left(\begin{array}{ccc}
1 & 0 & 0 \\
C_{2}^{2} & -2 C_{2} S_{2} & S_{2}^{2} \\
C_{3}^{2} & -2 C_{3} S_{3} & S_{3}^{2}
\end{array}\right)
$$

The $3 \times 3$ extended transfer matrix between monitors 1 and $i$ is given by

$$
\mathcal{T}_{i}=\left(\begin{array}{ccc}
C_{i} & S_{i} & \xi_{i} \\
C_{i}^{\prime} & S_{i}^{\prime} & \xi_{i}^{\prime} \\
0 & 0 & 1
\end{array}\right)
$$


where $\xi_{i}$ is the contribution to the dispersion due to a dipole magnet between the first and the $i$ th monitor. The propagation of the dispersion $D_{i}$ in terms of $D_{1}$ and its derivative $D_{1}^{\prime}$ is given by the expression

$$
D_{i}=C_{i} D_{1}+S_{i} D_{1}^{\prime}+\xi_{i} \quad i=2,3
$$

Using $\beta_{1} \gamma_{1}-\alpha_{1}^{2}=1$, the solution $\Sigma^{\prime}=\mathcal{M}^{-1} \Sigma$ of Eq. (4) can be cast into the form

$$
\begin{aligned}
& \beta_{1}=A / \sqrt{A C-B^{2}} \quad A=\Sigma_{1}^{\prime} \\
& \alpha_{1}=B / \sqrt{A C-B^{2}} \quad \text { with } \quad B=\Sigma_{2}^{\prime} \\
& \epsilon=\sqrt{A C-B^{2}} \quad C=\Sigma_{3}^{\prime}
\end{aligned}
$$

or, equivalently

$$
\epsilon=\sigma_{\beta, 1}^{2} \Lambda \quad \beta_{1}=\frac{1}{\Lambda} \quad \alpha_{1}=\frac{\Gamma}{2 \Lambda} .
$$

$\Gamma$ and $\Lambda$ are given in terms of the betatron profile widths $\sigma_{\beta, i}$

$$
\begin{aligned}
\Gamma & =\frac{\left[\left(\sigma_{\beta, 3} / \sigma_{\beta, 1}\right)^{2}-C_{3}^{2}\right] / S_{3}^{2}-\left[\left(\sigma_{\beta, 2} / \sigma_{\beta, 1}\right)^{2}-C_{2}^{2}\right] / S_{2}^{2}}{\left(C_{2} / S_{2}\right)-\left(C_{3} / S_{3}\right)} \\
\Lambda^{2} & =\left(\sigma_{\beta, 2} / \sigma_{\beta, 1}\right)^{2} / S_{2}^{2}-\left(C_{2} / S_{2}\right)^{2}+\left(C_{2} / S_{2}\right) \Gamma-\Gamma^{2} / 4 .
\end{aligned}
$$

The three quantities $\Sigma_{i}^{\prime}$ stand for the three components of the vector $\Sigma^{\prime}$.

In analogy with the approach based on five or more monitors, to be described below, $\alpha_{1}$, $\beta_{1}$ and $\epsilon$ may be expressed from Eqs. (3) and (7) using a $6 \times 6$ matrix formulation equivalent to Eq. (4). Provided $D_{1}, D_{1}^{\prime}$ and $\delta$ are known, one can write

$$
\Sigma=\mathcal{M} \Pi
$$

in terms of the new six-dimensional vectors $\Sigma$ and $\Pi$

$$
\boldsymbol{\Sigma}=\left(\begin{array}{c}
\sigma_{1}^{2} \\
\sigma_{2}^{2} \\
\sigma_{3}^{2} \\
D_{1} \delta^{2} \\
D_{1}^{\prime} \delta^{2} \\
\delta^{2}
\end{array}\right) \quad \boldsymbol{\Pi}=\left(\begin{array}{c}
\beta_{1} \epsilon+D_{1}^{2} \delta^{2} \\
\alpha_{1} \epsilon-D_{1} D_{1}^{\prime} \delta^{2} \\
\gamma_{1} \epsilon+D_{1}^{\prime 2} \delta^{2} \\
D_{1} \delta^{2} \\
D_{1}^{\prime} \delta^{2} \\
\delta^{2}
\end{array}\right)
$$

and the $6 \times 6$ matrix

$$
\mathcal{M}=\left(\begin{array}{cccccc}
1 & 0 & 0 & 0 & 0 & 0 \\
C_{2}^{2} & -2 C_{2} S_{2} & S_{2}^{2} & 2 C_{2} \xi_{2} & 2 S_{2} \xi_{2} & \xi_{2} \\
C_{3}^{2} & -2 C_{3} S_{3} & S_{3}^{2} & 2 C_{3} \xi_{3} & 2 S_{3} \xi_{3} & \xi_{3} \\
0 & 0 & 0 & 1 & 0 & 0 \\
0 & 0 & 0 & 0 & 1 & 0 \\
0 & 0 & 0 & 0 & 0 & 1
\end{array}\right) .
$$

The value of the derivative of the dispersion function $D^{\prime}{ }_{1}$ at the first monitor can be obtained from the knowledge of the measured dispersion $D_{i}$ at the other monitors using Eq. (7), yielding

$$
D_{1}^{\prime}=\frac{C_{3}\left(D_{2}-\xi_{2}\right)-C_{2}\left(D_{3}-\xi_{3}\right)}{C_{2} S_{3}-C_{3} S_{2}}
$$


The solution of Eq. (10) is given by $\Sigma^{\prime}=\mathcal{M}^{-1} \Sigma$, from which, the emittance and the Twiss parameters can be derived as

$$
\begin{aligned}
& \beta_{1}=A / \sqrt{A C-B^{2}} \quad A=\Sigma_{1}^{\prime}-D_{1}^{2} \delta^{2} \\
& \alpha_{1}=B / \sqrt{A C-B^{2}} \quad \text { with } \quad B=\Sigma_{2}^{\prime}+D_{1} D_{1}^{\prime} \delta^{2} \\
& \epsilon=\sqrt{A C-B^{2}} \quad C=\Sigma_{3}^{\prime}-D_{1}^{\prime 2} \delta^{2} \text {. }
\end{aligned}
$$

According to Eqs. (4) to (5) the presence of dipole magnets in the section of the beam line delimited by the first and last monitors has no influence on the determination of $\alpha_{1}, \beta_{1}$ and $\epsilon$.

\subsection{Method using five monitors}

The dispersion function $D$ can be determined experimentally at each monitor location by measuring the beam position for different beam momentum values and fitting the data using a linear model: the slope of the fitted straight line at each monitor represents the value of the dispersion function.

Although the measurement of the dispersion is conceptually simple, it requires the modification of some machine parameters and this is not always possible or easy to do during routine operation. However, from simple arguments, the information extracted from five monitors should be sufficient to compute the five unknown quantities $\alpha, \beta, D, D^{\prime} \epsilon$ (the relative momentum spread $\delta$ has to be known anyway). It turns out that this is the case, provided at least two dipoles are installed in the section of the beam line between the first and last monitor.

Once again the starting point is Eq. (1), connecting the optical parameters and the measured beam profiles in the presence of dispersion, and the transformation rules (3) and (7) used to propagate the Twiss parameters and the dispersion. Eq. (1) can be cast in the form of a system of five equations $\Sigma=\mathcal{M} \Pi$ similar to Eq. (4), by introducing the five-dimensional vectors $\Sigma$ and $\Pi$

$$
\boldsymbol{\Sigma}=\left(\begin{array}{c}
\sigma_{1}^{2} \\
\sigma_{2}^{2}-\xi_{2}^{2} \delta^{2} \\
\sigma_{3}^{2}-\xi_{3}^{2} \delta^{2} \\
\sigma_{4}^{2}-\xi_{4}^{2} \delta^{2} \\
\sigma_{5}^{2}-\xi_{5}^{2} \delta^{2}
\end{array}\right) \quad \boldsymbol{\Pi}=\left(\begin{array}{c}
\beta_{1} \epsilon+D_{1}^{2} \delta^{2} \\
\alpha_{1} \epsilon-D_{1} D_{1}^{\prime} \delta^{2} \\
\gamma_{1} \epsilon+D_{1}^{\prime 2} \delta^{2} \\
D_{1} \delta^{2} \\
D_{1}^{\prime} \delta^{2}
\end{array}\right)
$$

and the $5 \times 5$ matrix

$$
\mathcal{M}=\left(\begin{array}{ccccc}
1 & 0 & 0 & 0 & 0 \\
C_{2}^{2} & -2 C_{2} S_{2} & S_{2}^{2} & 2 C_{2} \xi_{2} & 2 S_{2} \xi_{2} \\
C_{3}^{2} & -2 C_{3} S_{3} & S_{3}^{2} & 2 C_{3} \xi_{3} & 2 S_{3} \xi_{3} \\
C_{4}^{2} & -2 C_{4} S_{4} & S_{4}^{2} & 2 C_{4} \xi_{4} & 2 S_{4} \xi_{4} \\
C_{5}^{2} & -2 C_{5} S_{5} & S_{5}^{2} & 2 C_{5} \xi_{5} & 2 S_{5} \xi_{5}
\end{array}\right) .
$$

A necessary condition for the matrix $\mathcal{M}$ to be invertible (i.e. non singular) is that at least two quantities $\xi_{i}$ are different from zero.

Provided the matrix $\mathcal{M}$ can be inverted, the solution is given by $\Sigma^{\prime}=\mathcal{M}^{-1} \Sigma$ and the unknown parameters can be expressed as follows

$$
\begin{aligned}
& D_{1}=\quad \Sigma_{4}^{\prime} / \delta^{2} \\
& D_{1}^{\prime}=\Sigma_{5}^{\prime} / \delta^{2} \\
& \beta_{1}=A / \sqrt{A C-B^{2}} \quad A=\Sigma_{1}^{\prime}-\Sigma_{4}^{\prime 2} / \delta^{2} \\
& \alpha_{1}=B / \sqrt{A C-B^{2}} \quad \text { with } \quad B=\Sigma_{2}^{\prime}+\Sigma_{4}^{\prime} \Sigma_{5}^{\prime} / \delta^{2} \\
& \epsilon=\sqrt{A C-B^{2}} \quad C=\Sigma_{3}^{\prime}-\Sigma_{5}^{\prime 2} / \delta^{2} \text {. }
\end{aligned}
$$


Similarly, the system of equations (15) and (16) may be recast in a $6 \times 6$ matrix form in which the vectors $\Sigma$ and $\Pi$ are given by

$$
\boldsymbol{\Sigma}=\left(\begin{array}{c}
\sigma_{1}^{2} \\
\sigma_{2}^{2} \\
\sigma_{3}^{2} \\
\sigma_{4}^{2} \\
\sigma_{5}^{2} \\
\delta^{2}
\end{array}\right) \quad \boldsymbol{\Pi}=\left(\begin{array}{c}
\beta_{1} \epsilon+D_{1}^{2} \delta^{2} \\
\alpha_{1} \epsilon-D_{1} D_{1}^{\prime} \delta^{2} \\
\gamma_{1} \epsilon+D_{1}^{\prime 2} \delta^{2} \\
D_{1} \delta^{2} \\
D_{1}^{\prime} \delta^{2} \\
\delta^{2}
\end{array}\right)
$$

$\mathcal{M}$ takes the form

$$
\mathcal{M}=\left(\begin{array}{cccccc}
1 & 0 & 0 & 0 & 0 & 0 \\
C_{2}^{2} & -2 C_{2} S_{2} & S_{2}^{2} & 2 C_{2} \xi_{2} & 2 S_{2} \xi_{2} & \xi_{2}^{2} \\
C_{3}^{2} & -2 C_{3} S_{3} & S_{3}^{2} & 2 C_{3} \xi_{3} & 2 S_{3} \xi_{3} & \xi_{3}^{2} \\
C_{4}^{2} & -2 C_{4} S_{4} & S_{4}^{2} & 2 C_{4} \xi_{4} & 2 S_{4} \xi_{4} & \xi_{4}^{2} \\
C_{5}^{2} & -2 C_{5} S_{5} & S_{5}^{2} & 2 C_{5} \xi_{5} & 2 S_{5} \xi_{5} & \xi_{5}^{2} \\
0 & 0 & 0 & 0 & 0 & 1
\end{array}\right)
$$

and the solution is still $\Sigma^{\prime}=\mathcal{M}^{-1} \Sigma$. In Appendix A the extension of the five-monitor method to the case of a periodic transport channel is presented.

\subsection{Method using six monitors}

If six monitors are installed in a transfer line, the application of the method described in the previous Section to the different combinations of five monitors out of the six available could be considered. Then, the computed values of the optical parameters could be averaged thus reducing systematic errors. Another possibility would be to select the best set of five monitors out of the whole ensemble and to apply the technique to such a subset.

A different approach is to use the information provided by the six monitors to evaluate not only the optical parameters $\alpha_{1}, \beta_{1}, D_{1}, D_{1}^{\prime}$ and $\epsilon$, but also the momentum spread $\delta$ which was assumed to be known in the previous Section. The arguments used to derive the previous equations can be adapted to this situation. Eq. (4) is still valid, but now the vectors $\Sigma$ and $\Pi$ have dimension six i.e.

$$
\boldsymbol{\Sigma}=\left(\begin{array}{c}
\sigma_{1}^{2} \\
\sigma_{2}^{2} \\
\sigma_{3}^{2} \\
\sigma_{4}^{2} \\
\sigma_{5}^{2} \\
\sigma_{6}^{2}
\end{array}\right) \quad \boldsymbol{\Pi}=\left(\begin{array}{c}
\beta_{1} \epsilon+D_{1}^{2} \delta^{2} \\
\alpha_{1} \epsilon-D_{1} D^{\prime}{ }_{1} \delta^{2} \\
\gamma_{1} \epsilon+D^{\prime 2}{ }_{1}^{2} \\
D_{1} \delta^{2} \\
D^{\prime}{ }_{1}^{2} \delta^{2} \\
\delta^{2}
\end{array}\right)
$$

while $\mathcal{M}$ is

$$
\mathcal{M}=\left(\begin{array}{cccccc}
1 & 0 & 0 & 0 & 0 & 0 \\
C_{2}^{2} & -2 C_{2} S_{2} & S_{2}^{2} & 2 C_{2} \xi_{2} & 2 S_{2} \xi_{2} & \xi_{2}^{2} \\
C_{3}^{2} & -2 C_{3} S_{3} & S_{3}^{2} & 2 C_{3} \xi_{3} & 2 S_{3} \xi_{3} & \xi_{3}^{2} \\
C_{4}^{2} & -2 C_{4} S_{4} & S_{4}^{2} & 2 C_{4} \xi_{4} & 2 S_{4} \xi_{4} & \xi_{4}^{2} \\
C_{5}^{2} & -2 C_{5} S_{5} & S_{5}^{2} & 2 C_{5} \xi_{5} & 2 S_{5} \xi_{5} & \xi_{5}^{2} \\
C_{6}^{2} & -2 C_{6} S_{6} & S_{6}^{2} & 2 C_{6} \xi_{6} & 2 S_{6} \xi_{6} & \xi_{6}^{2}
\end{array}\right)
$$


The necessary condition for $\mathcal{M}$ to be invertible is that at least three quantities $\xi_{i}$ are different from zero.

Whenever the conditions for the matrix $\mathcal{M}$ to be non-singular are fulfilled, the optical parameters can be computed by using the following expressions

$$
\begin{aligned}
& \delta^{2}=\quad \Sigma_{6}^{\prime} \\
& D_{1}=\Sigma_{4}^{\prime} / \Sigma_{6}^{\prime} \\
& D_{1}^{\prime}=\Sigma_{5}^{\prime} / \Sigma_{6}^{\prime} \\
& \beta_{1}=A / \sqrt{A C-B^{2}} \quad A=\Sigma_{1}^{\prime}-\Sigma_{4}^{\prime 2} / \Sigma_{6}^{\prime} \\
& \alpha_{1}=B / \sqrt{A C-B^{2}} \quad \text { with } \quad B=\Sigma_{2}^{\prime}+\Sigma_{4}^{\prime} \Sigma_{5}^{\prime} / \Sigma_{6}^{\prime} \\
& \epsilon=\sqrt{A C-B^{2}} \quad C=\Sigma_{3}^{\prime}-\Sigma_{5}^{\prime 2} / \Sigma_{6}^{\prime} \text {. }
\end{aligned}
$$

\subsection{Method using more than six monitors}

When more than six monitors are installed in the transfer line it is possible to choose between two different approaches: the first one consists of the application of the deterministic equations obtained in the previous Sections to a subset of five or six monitors.

A second technique is based on a fit procedure: the model expressed by Eqs. (3), (7) is used to find the best parameters that match the beam widths measured on the different monitors. For this second technique, the starting point is the definition of a function to be minimised. The natural choice is the following function

$$
\chi^{2}(\boldsymbol{\Pi})=\sum_{i=1}^{N_{\mathrm{mon}}}\left[\frac{y_{i}-\bar{y}_{i}(\boldsymbol{\Pi})}{\Delta_{i}}\right]^{2},
$$

where $N_{\text {mon }}$ is the number of monitors, $y_{i}$ represents the square of the beam width measured on the $i$ th monitor, $\bar{y}_{i}(\boldsymbol{\Pi})$ is the square of the beam-width expressed in terms of the transfer matrix elements $C_{i}, S_{i}, \xi_{i}$ and the components of the vector $\Pi$, defined in a similar way to Eqs. (15) or (20) depending on whether the momentum spread is known or unknown. Finally, $\Delta_{i}$ stands for the measurement error associated with $y_{i}$.

The goal is to minimise the function $\chi^{2}$ and this is achieved by solving the following equations

$$
\frac{\partial \chi^{2}(\boldsymbol{\Pi})}{\partial \Pi_{i}}=0 \quad 1 \leq i \leq 5 \text { or } 6
$$

After some algebra, it is possible to recast Eq. (24) in the following form similar to Eq. (4)

$$
\bar{\Sigma}=\overline{\mathcal{M}} \Pi
$$

Here $\bar{\Sigma}$ and $\overline{\mathcal{M}}$ are similar to the vector $\Sigma$ and to the matrix $\mathcal{M}$ defined in Eqs. (20) and (21), but with the $i$ th line rescaled by the quantity $\Delta_{i}$, i.e. $\bar{\Sigma}=\Delta \Sigma$ and $\overline{\mathcal{M}}=\Delta \mathcal{M}$, with the

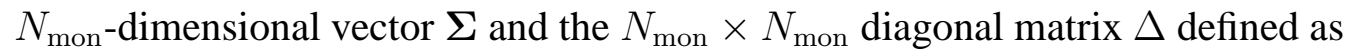

$$
\boldsymbol{\Sigma}=\left(\begin{array}{c}
\sigma_{1}^{2} \\
\sigma_{2}^{2} \\
\vdots \\
\sigma_{\text {mon }}^{2}
\end{array}\right) \quad \Delta=\left(\begin{array}{cccc}
\Delta_{1}^{-1} & 0 & \cdots & 0 \\
0 & \Delta_{2}^{-1} & \cdots & 0 \\
\vdots & \vdots & \vdots & \vdots \\
0 & 0 & \cdots & \Delta_{\text {mon }}^{-1}
\end{array}\right)
$$


If the momentum spread is unknown, then, $\mathcal{M}$ is represented by a rectangular $N_{\text {mon }} \times 6$ matrix of the form

$$
\mathcal{M}=\left(\begin{array}{cccccc}
1 & 0 & 0 & 0 & 0 & 0 \\
C_{2}^{2} & -2 C_{2} S_{2} & S_{2}^{2} & 2 C_{2} \xi_{2} & 2 S_{2} \xi_{2} & \xi_{2}^{2} \\
\vdots & \vdots & \vdots & \vdots & \vdots & \vdots \\
C_{\text {mon }}^{2} & -2 C_{\text {mon }} S_{\text {mon }} & S_{\text {mon }}^{2} & 2 C_{\text {mon }} \xi_{\text {mon }} & 2 S_{\text {mon }} \xi_{\text {mon }} & \xi_{\text {mon }}^{2}
\end{array}\right) .
$$

Provided $\overline{\mathcal{M}}^{t} \overline{\mathcal{M}}$ is non-singular, the solution $\Sigma^{\prime}$ of Eq. (25) can be written as

$$
\Sigma^{\prime}=\left(\overline{\mathcal{M}}^{t} \overline{\mathcal{M}}\right)^{-1} \overline{\mathcal{M}}^{t} \bar{\Sigma}
$$

where $\overline{\mathcal{M}}^{t}$ is the transpose matrix of $\overline{\mathcal{M}}$.

The optical parameters and the momentum spread can be found as in Eq. (22). The reduction of this method to the case where the momentum spread is known is straightforward, i.e. the last column of $\mathcal{M}$ and the last line of $\Pi$ must be dropped, yielding a $N_{\text {mon }} \times 5$ matrix and a five-dimensional vector, respectively. Furthermore, Eq. (17) should be applied in place of Eq. (22) to obtain the optical parameters.

It can be verified that this approach is equivalent to the deterministic solution presented in the previous Sections when $N_{\text {mon }}=3,5,6$.

\subsection{Error estimates}

Under the assumption that the errors on the measured beam profiles are statistically independent, the error on the fit parameters $\operatorname{err}\left(\Sigma_{i}^{\prime}\right)$ is given by

$$
\operatorname{err}^{2}\left(\Sigma_{i}^{\prime}\right)=\sum_{l=1}^{N_{\text {mon }}}\left(\frac{\partial \Sigma_{i}^{\prime}}{\partial y_{l}}\right)^{2} \Delta_{l}^{2}
$$

Introducing for convenience the matrix $\mathcal{B}=\left(\overline{\mathcal{M}}^{t} \overline{\mathcal{M}}\right)^{-1}$, Eqs. (28-29) yield

$$
\frac{\partial \Sigma_{i}^{\prime}}{\partial y_{l}}=\sum_{j=1}^{6} \mathcal{B}_{i, j} \frac{\mathcal{M}_{j, l}^{t}}{\Delta_{l}^{2}}
$$

and

$$
\sum_{l=1}^{N_{\text {mon }}}\left(\frac{\partial \Sigma_{i}^{\prime}}{\partial y_{l}}\right)^{2} \Delta^{2}{ }_{l}=\sum_{j=1}^{6} \sum_{k=1}^{6} \sum_{l=1}^{N_{\text {mon }}} \mathcal{B}_{i, j} \mathcal{B}_{i, k} \frac{\mathcal{M}_{j, l}^{t} \mathcal{M}_{l, k}}{\Delta_{l}^{2}}=\sum_{j=1}^{6} \sum_{k=1}^{6} \mathcal{B}_{i, k} \mathcal{B}_{i, j} \mathcal{B}_{j, k}^{-1}
$$

from which the final result is obtained

$$
\operatorname{err}^{2}\left(\Sigma_{i}^{\prime}\right)=\mathcal{B}_{i, i}
$$

The matrix $\mathcal{B}$ is in fact, the covariant matrix and its diagonal elements provide an estimate of the error on the fitted parameters.

The error on the measured Twiss parameters can be deduced from (32) by applying the standard techniques of error propagation to the relations (17) and (22). Note that the condition $\operatorname{det}\left(\overline{\mathcal{M}}^{t} \overline{\mathcal{M}}\right) \neq 0$ together with the minimisation of the terms $\mathcal{B}_{i, i}$ can be used to determine the optimal monitor locations to derive the optical and beam parameters according to the new methods derived in previous Sections. 


\section{Application of the new methods to derive optical and beam parameters}

\subsection{The model}

The approach described in the previous Sections has been applied to the transfer line between the CERN Proton Synchrotron (PS) and the Super Proton Synchrotron (SPS). This line is divided into two consecutive parts: the TT2 line and the TT10 line. At the junction of the two lines, the beam is deflected $\approx 81 \mathrm{mrad}$ to the right. Due to the difference in height between the PS and SPS, a vertical deflection angle of $\approx 60 \mathrm{mrad}$ is also imposed at the entrance of TT10 and then cancelled before injection in the SPS.

Three Secondary Emission Monitors (SEMs) are installed in each the TT2 and TT10 section. These two sets of monitors are routinely used to perform emittance and Twiss parameter measurement in both lines. For this purpose, the standard method based on Eq. (9) is used, with the dispersion measured by performing an energy shift.

In addition, four Optical Transition Radiation screens (OTRs) [11, 12] are installed in the TT10 transfer line. They also allow the measurement of beam profiles, but they have the advantage of a better resolution, of providing a three dimensional view of the beam distribution and of inducing a negligible blow-up on the beam.

Fig. 1 shows the most relevant optical parameters of the TT2/TT10 transfer line. In the upper part the horizontal and vertical $\beta$-functions are shown, in the centre the horizontal and vertical dispersion functions are plotted, while in the lower part the horizontal and vertical beam envelopes are shown. Also marked are the positions of the ten beam profile monitors. The optical parameters shown refer to the transfer line setting to be used for the future LHC operation.

\subsection{Results of numerical simulations}

A series of numerical simulations have been carried out to test the performance of the different methods presented. The aim of such simulations is twofold: Firstly, using Eq. (32), the problem of optimising the position of three monitors so that the accuracy of the measurement is maximised has been considered. Secondly, a comparison of the accuracy of the various methods presented in the previous Sections has been carried out. The main point of this analysis consists of the determination of the behaviour of the computed optical parameters with their dependence on measurement errors affecting the beam profile width $\sigma_{i}$.

\subsubsection{Optimisation of the monitor positions}

For these numerical simulations, the starting point defines a target function to be minimised. Following the analysis carried out in the previous Section, it is quite natural to define this target function as the sum of the errors on the parameters $\Sigma_{i}^{\prime}$ :

$$
\sum_{i=1}^{6} \operatorname{err}^{2}\left(\Sigma_{i}^{\prime}\right)=\sum_{i=1}^{6} \mathcal{B}_{i, i}=\operatorname{tr} \mathcal{B}
$$

Using the standard parametrisation of the transfer matrix in terms of Twiss parameters, one can express the trace of the matrix $\mathcal{B}$ as

$$
\operatorname{tr} \mathcal{B}=\operatorname{tr} \mathcal{B}\left(\Delta \varphi_{2}, \Delta \varphi_{3}\right)
$$

where $\Delta \varphi_{i}$ represents the phase advance between the $i$ th monitor and the first one. The optimisation has been applied to the three-monitors method. By using the MAD program [13], the transfer matrices $\mathcal{T}_{i}$ have been computed at a selected number of locations along the transfer line, then the function $\operatorname{tr} \mathcal{B}$ has been evaluated. The location of the first monitor has been kept 

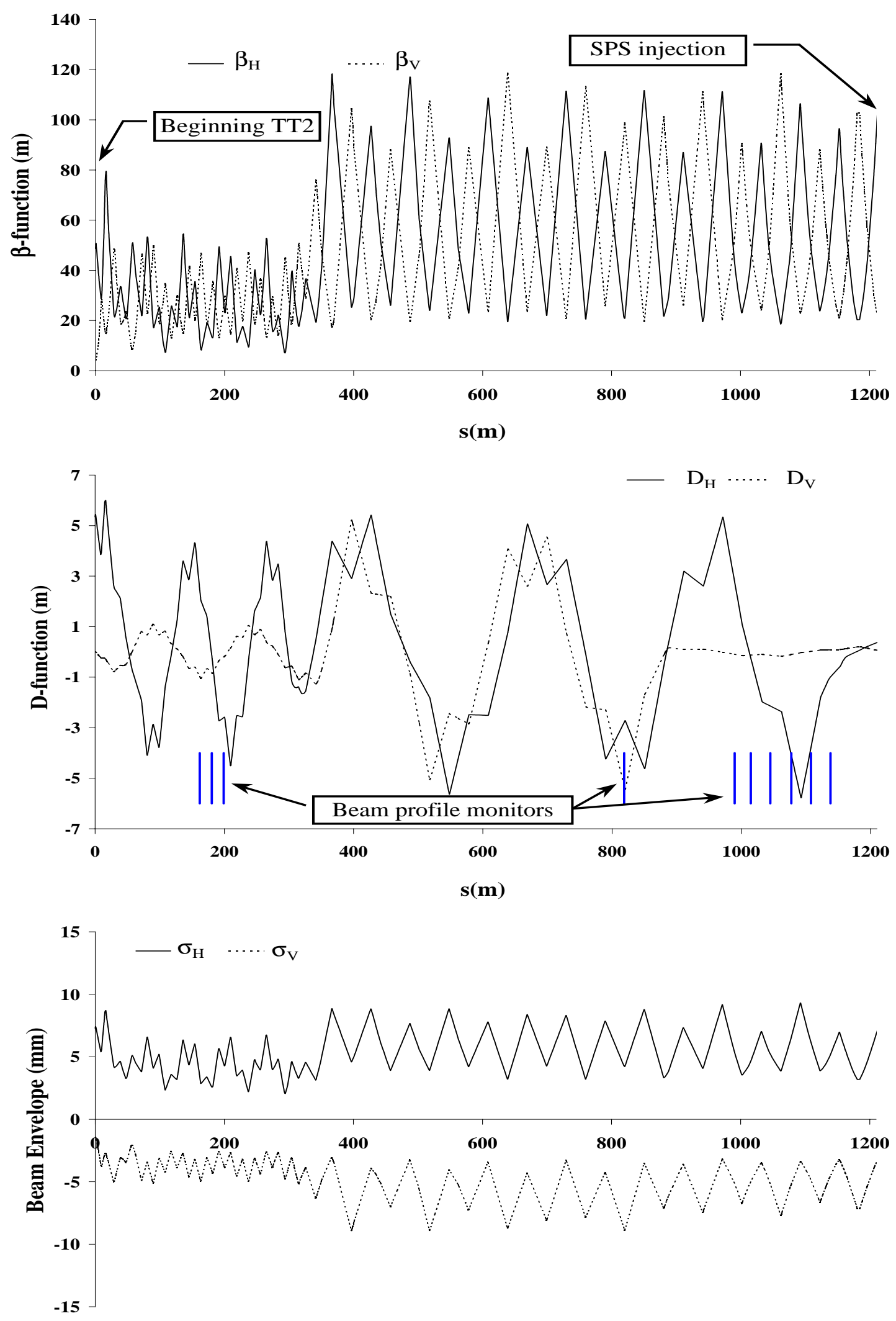

$\mathbf{s}(\mathbf{m})$

Figure 1: Horizontal and vertical $\beta$-functions (upper part), dispersion function (centre part) and beam envelope (lower part) for the TT2/TT10 line for rms emittance of $\epsilon_{\mathrm{H}}=\epsilon_{\mathrm{V}}=0.25 \mu \mathrm{m}$ and rms beam's momentum spread of $1.0 \times 10^{-3}$. The position of the beam profile monitors installed in the transfer lines is also shown. 

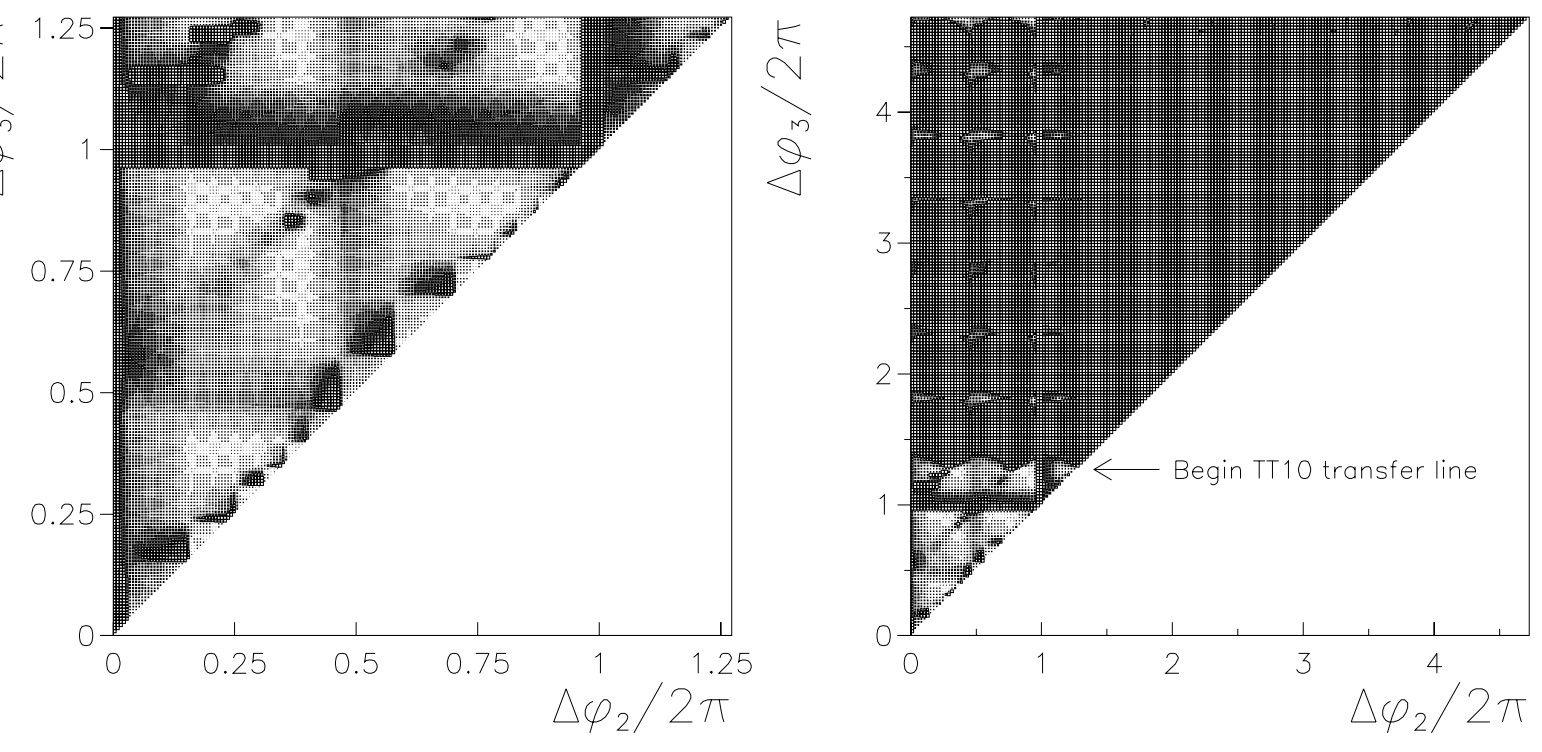

Figure 2: Two-dimensional plots of $\operatorname{tr} \mathcal{B}$ as a function of $\Delta \varphi_{2}, \Delta \varphi_{3}$. On the left part the plot is restricted to the TT2 section, while in the right part the function over the whole transfer line is shown. The colour is proportional to the function value: higher values correspond to darker regions. Hence, local and global minima correspond to lighter (white) regions.

fixed, while the position of the remaining two monitors has been varied along the transfer line. The results are shown in Fig. 2. The surface representing the graph of $\operatorname{tr} \mathcal{B}$ has been projected on a 2D plot: in the left part a plot of the function restricted to the TT2 section is shown, while in the right part $\operatorname{tr} \mathcal{B}$ over the whole TT2/TT10 transfer line is shown. The colour is proportional to the function value: higher values correspond to darker regions. Hence, local and global minima correspond to lighter (white) regions. The results refer to the horizontal plane. Some features are apparent:

- The triangular shape of the plots is due to the fact that in the simulations the order of the monitors is never reversed, hence the following holds $\Delta \varphi_{3}>\Delta \varphi_{2}$.

- The configurations having $\Delta \varphi_{i}=2 k \pi$ represent 'bad' configurations. This can be easily understood as, in this case, two out of the three monitors are actually equivalent as far as the optical conditions are concerned.

- Configurations having both the second and the third monitor in the TT10 transfer line are 'bad'.

- Some configurations having only one monitor in the TT10 transfer line correspond to local minima of the function $\operatorname{tr} \mathcal{B}$. By comparing the results with the optical functions shown in Fig. 1 it is found that the local minima correspond to locations where the horizontal beta-function is maximum.

- A direct inspection of the numerical values of $\operatorname{tr} \mathcal{B}$ allows to determine that the global minimum is attained for $\Delta \varphi_{2} / 2 \pi=0.405$ and $\Delta \varphi_{3} / 2 \pi=0.781$, i.e. when all the three monitors are placed in the TT2 transfer line. The value of the phase advance in the horizontal plane for the actual position of the monitors is $\Delta \varphi_{2} / 2 \pi=0.236$ and $\Delta \varphi_{3} / 2 \pi=0.347$, corresponding to a local minimum of the function $\operatorname{tr} \mathcal{B}$. It is worthwhile stressing the fact that the optimal configuration does not obey the common rule stating that the phase-advance must be $\Delta \varphi_{2}=1 / 3$ and $\Delta \varphi_{3}=2 / 3$. 


\subsubsection{Comparison of the accuracy of the different methods}

Using nominal values for the initial optical parameters $\alpha, \beta, D, D^{\prime}, \epsilon, \delta$, the corresponding values of $\sigma_{i}$ at the monitor locations have been computed, thus generating a sequence $\mathcal{S}=\left\{\sigma_{i}\right\}_{i=1, N_{\text {mon }}}$. In the numerical simulations it has been assumed that the error affecting the transfer matrices is negligible with respect to the error on the beam profile due to the random fluctuations of the beam characteristics and the accuracy of the monitors. To simulate the effect of measurement errors and fluctuations in the beam properties new sequences have been derived by the following process

$$
\bar{\sigma}_{i}(j)=\sigma_{i}\left[1+\kappa \eta_{i}(j)\right], \quad i=1, N_{\text {mon }}, \quad j=1, N_{\text {set }},
$$

where $\eta_{i}(j)$ represents a Gaussian random variable with mean 0 and sigma 1 . The parameter $\kappa$ in Eq. (35) represents the relative error on $\bar{\sigma}_{i}$. From a number of tests performed in the TT2/TT10 transfer line, it has been decided to use $\kappa=10^{-2}$ in the numerical simulations. As a result of the application of Eq. (35), $N_{\text {set }}$ sequences $\overline{\mathcal{S}}_{j}=\left\{\bar{\sigma}_{i}(j)\right\}_{i=1, N_{\text {mon }}}$ are generated. In the numerical simulations presented in this paper, $N_{\text {set }}=5 \times 10^{4}$ has been used to have an accurate reconstruction of the distribution of the measured optical parameters.

In Fig. 3 the results of such simulations are given: the distribution of the Twiss parameters for the three-monitor method (shaded) as well as for the five-monitor approach are plotted. Similarly, the distribution of the beam emittance is shown in Fig. 4. When a smaller number
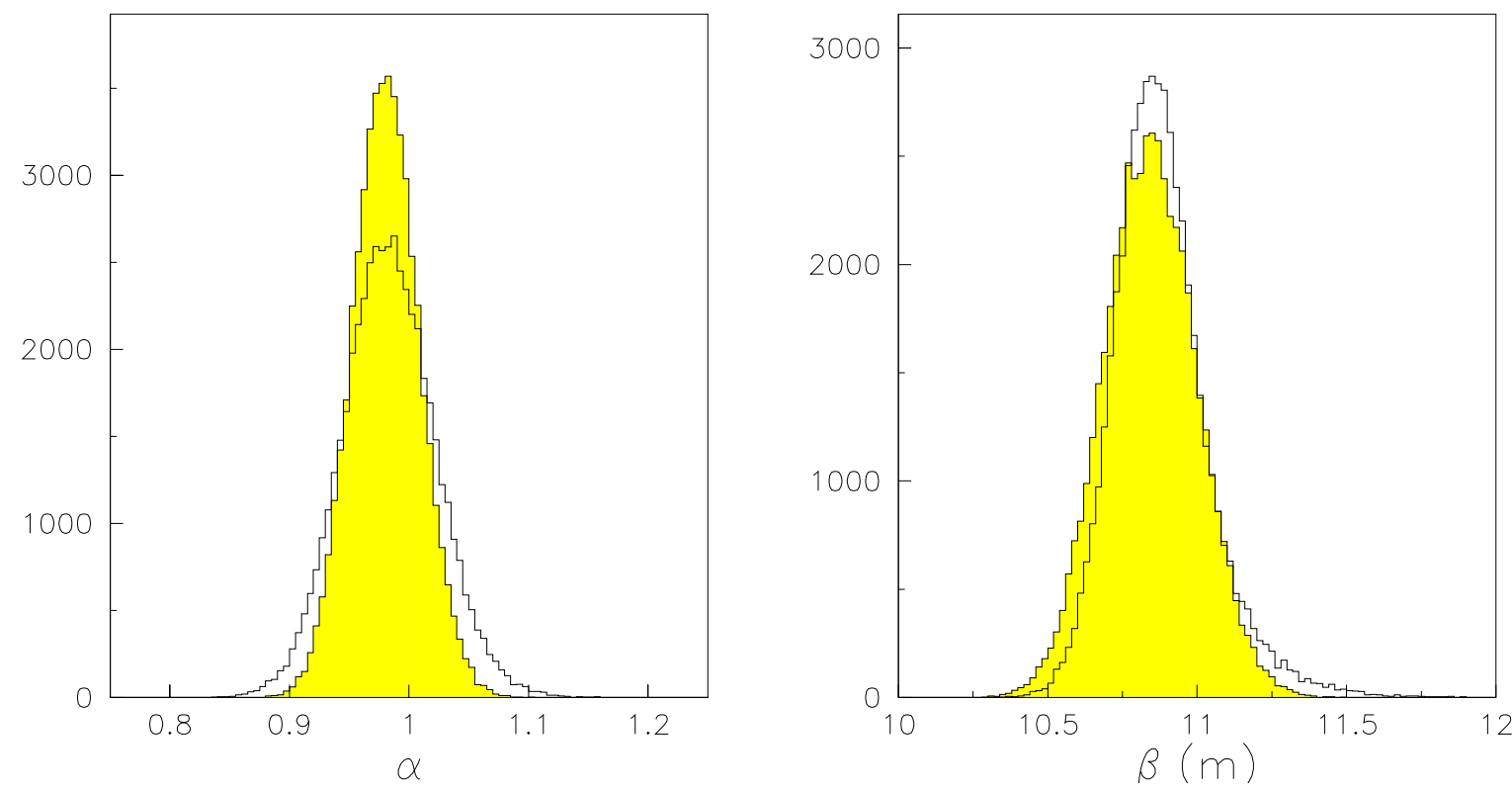

Figure 3: Distribution of the horizontal Twiss parameters $\alpha$ (left) and $\beta$ (right) at the location of the first monitor in TT2 as computed using the standard three-monitor method (shaded) or the five-monitor method.

of monitors than those available is used to compute the optical parameters, it has to be decided how to chose the best ones among the whole set. As far as the three-monitor method is concerned, it has been decided to use the first three monitors installed in the TT2 transfer line. In the case of the five-monitor approach, the choice has been based on the minimisation of the function $\operatorname{tr} \mathcal{B}$. This procedure allowed considerable improvement in the accuracy of the results. No difference is found in the outcome of the numerical computations: the three distributions presented in Figs. 3-4 are very similar as far as the overall shape, the mean value and the sigma 
are concerned. Similar results hold when the Twiss parameters are derived using the six-monitor method.

In Fig. 5 results for the dispersion function and its derivative are presented. In this case, the five-monitor method and the six-monitor method (shaded distribution) have been used.

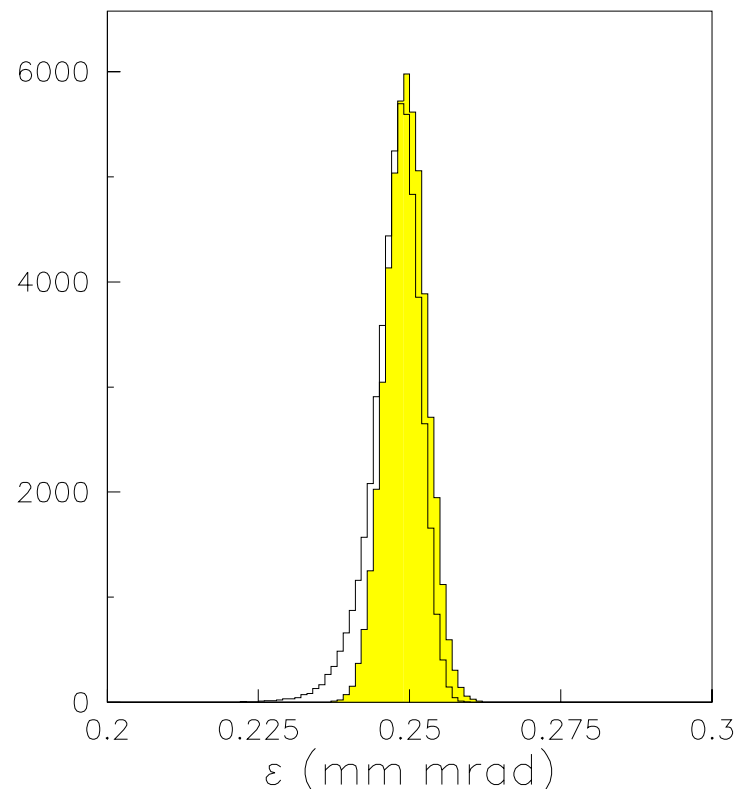

Figure 4: Distribution of the horizontal beam emittance $\epsilon$ computed using the standard threemonitor method (shaded) or the five-monitor method.
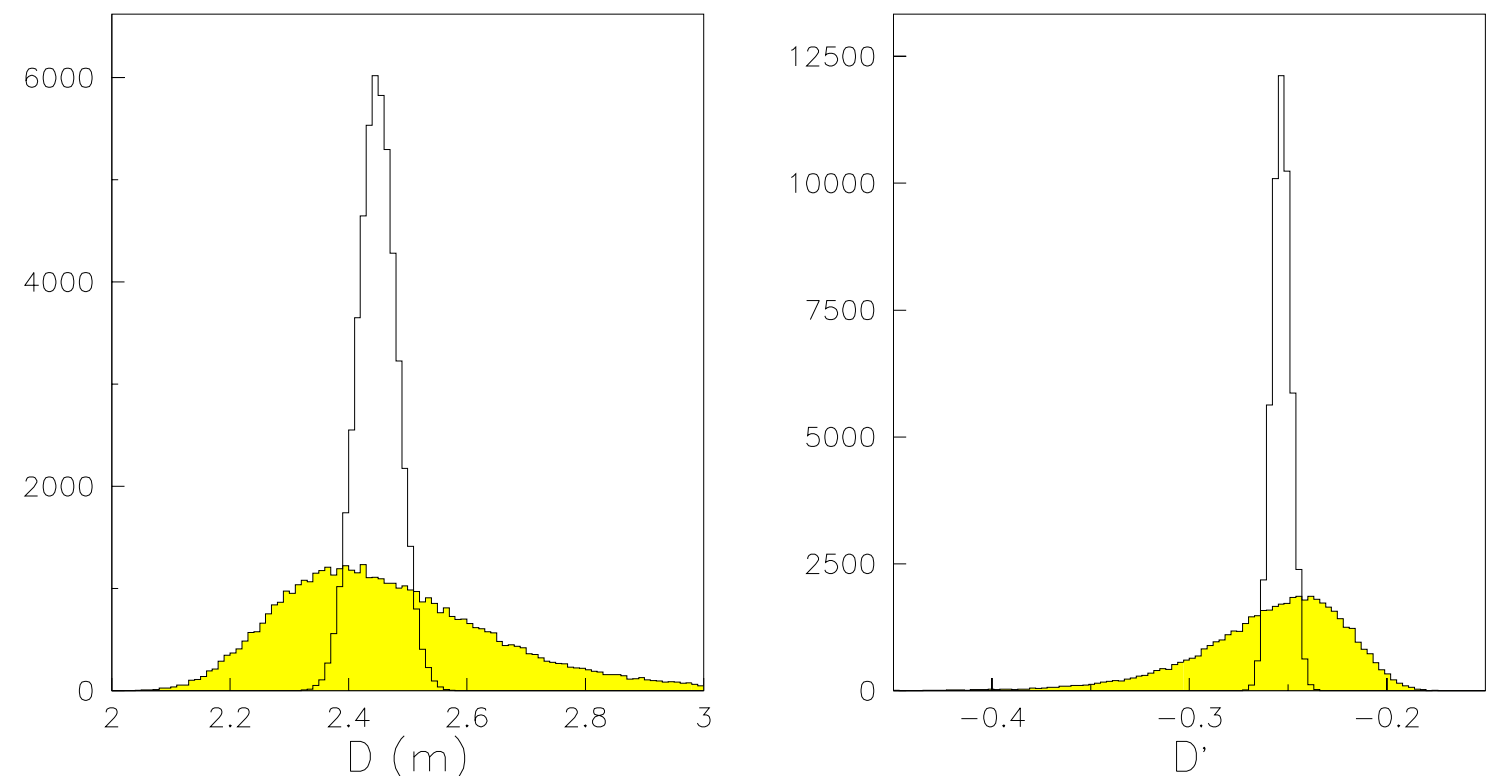

Figure 5: Distribution of the horizontal dispersion $D$ (left) and dispersion derivative $D^{\prime}$ (right) at the location of the first monitor in the TT2 line as computed using the five-monitor method or the six-monitor method (shaded).

Of course, the approach based on the three-monitor method cannot be considered in this comparison as it is based on the a-priori knowledge of the dispersion function value at the 


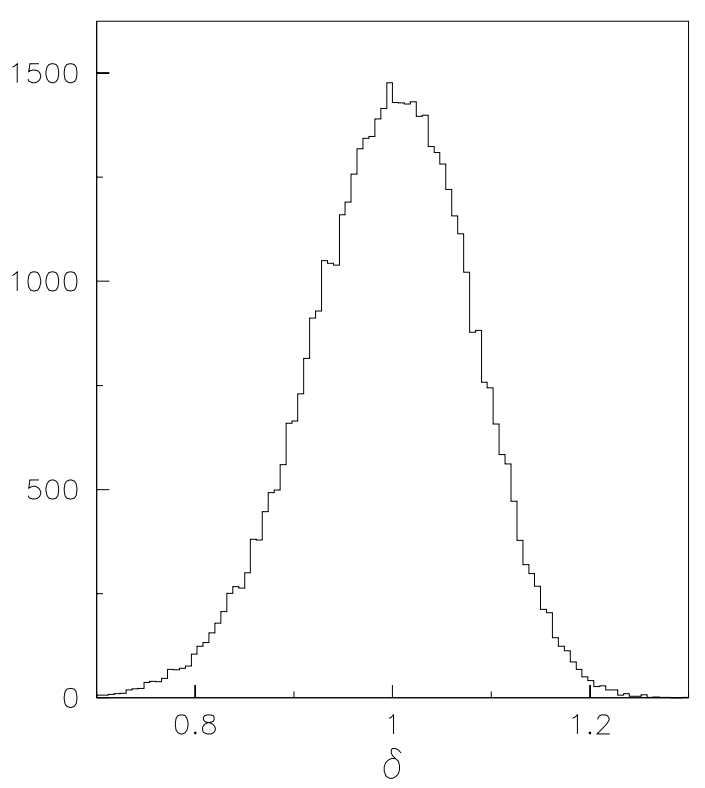

Figure 6: Distribution of the beam momentum spread $\delta$ (in units of $10^{-3}$ as computed using the six-monitor method.

The distributions of $D, D^{\prime}$ are somewhat different for the two approaches. The fivemonitor method generates distributions that resemble closely Gaussian distributions. This is a direct consequence of the form of Eqs. (17). The six-monitor method, however, produces skewed distributions with long tails. The explanation for this behaviour can be found by considering also the distribution of the beam's momentum spread computed using the six-monitors approach shown in Fig. 6. A careful analysis of Eqs. (22) shows that the method allows the direct computation of the product $D \delta^{2}$ or $D^{\prime} \delta^{2}$, therefore, any asymmetry in the distribution function of $\delta^{2}$ propagates also to $D, D^{\prime}$. In Table 1 the results obtained using the different methods are summarised. The errors associated with the different reconstructed optical or beam parameters are deduced from the sigmas of the distributions presented in Figs. 3-6.

A final test has been carried out, the three-, five- and six-monitor methods have been

\begin{tabular}{|l||c|c|c|r|}
\hline \hline & Nominal values & Three monitors & Five Monitors & \multicolumn{1}{|c|}{ Six monitors } \\
\hline \hline$\alpha$ & 0.98 & $0.97 \pm 0.04$ & $0.98 \pm 0.05$ & $0.98 \pm 0.10$ \\
$\beta$ & 10.84 & $10.85 \pm 0.15$ & $10.86 \pm 0.22$ & $10.88 \pm 0.40$ \\
$D$ & 2.45 & - & $2.45 \pm 0.03$ & $2.47 \pm 0.17$ \\
$D^{\prime}$ & -0.25 & - & $-0.25 \pm 0.05$ & $-0.26 \pm 0.04$ \\
$\epsilon$ & 0.25 & $0.248 \pm 0.003$ & $0.249 \pm 0.004$ & $0.244 \pm 0.009$ \\
$\delta$ & $1.0 \times 10^{-3}$ & - & - & $0.99 \pm 0.07$ \\
\hline \hline
\end{tabular}

Table 1: Optical parameters for the transfer line optics used in the simulations as computed using the various methods described in the previous Sections. The nominal values are also shown. The errors associated with the reconstructed parameters are obtained from the distributions presented in Figs. 3-6.

applied to a situation where $\delta$ is about ten time smaller than the value used in previous compu- 
tations. Under this condition, the influence of the dispersion function on the beam size is highly reduced thus making the task of deriving the whole set of five or six optical and beam parameters rather hard.

The six-monitor method is the most sensible to the value of $\delta$. In fact, it turned out that the actual location of the monitors in the TT2/TT10 line does not allow to apply the six-monitor method to this case and it has been necessary to optimise their position. Only the first monitor in the TT2 transfer line has been retained. The remaining five monitors have been repositioned to minimise the value of the function $\operatorname{tr} \mathcal{B}$. In Table 2 are summarised the results obtained with the different methods together with the nominal value of the parameters used in the simulations.

The three-monitor method is almost unaffected by the drastic reduction of $\delta$. As far as

\begin{tabular}{|l||c|c|c|r|}
\hline \hline & Nominal values & Three monitors & Five Monitors & \multicolumn{1}{|c|}{ Six monitors } \\
\hline \hline$\alpha$ & 0.98 & $0.98 \pm 0.03$ & $0.98 \pm 0.04$ & $0.98 \pm 0.05$ \\
$\beta$ & 10.84 & $10.84 \pm 0.16$ & $10.88 \pm 0.16$ & $10.86 \pm 0.49$ \\
$D$ & 2.45 & - & $2.45 \pm 0.77$ & $2.03 \pm 0.80$ \\
$D^{\prime}$ & -0.25 & - & $-0.26 \pm 0.14$ & $-0.18 \pm 0.25$ \\
$\epsilon$ & 0.25 & $0.249 \pm 0.003$ & $0.248 \pm 0.004$ & $0.25 \pm 0.15$ \\
$\delta$ & $0.13 \times 10^{-3}$ & - & - & $0.18 \pm 0.07$ \\
\hline \hline
\end{tabular}

Table 2: Optical parameters for the transfer line optics used in the simulations as computed using the various methods described in the previous Sections for the smaller value of the beam's momentum spread. The errors associated with the reconstructed parameters are derived from the distributions of the computed parameters.

the five-monitor method is concern, the main effect consists in an increase of the sigmas of the distribution functions of the reconstructed parameters. For the six-monitor method, however, both the mean values and the associated errors are affected. In particular, the average value of $\delta$ is higher than the nominal value, thus inducing a bias in the values of $D, D^{\prime}$. This is a good example of how the 'best beam' that fit experimental data does not always coincide with the 'real beam' as compensations between the various fit parameters can occur.

\section{Conclusions}

New techniques to compute the optical parameters in a transfer line from the knowledge of $N_{\text {mon }}$ beam profiles have been presented and discussed in detail. A general formula to evaluate the intrinsic accuracy of the various methods has been worked out. Furthermore, it has been shown how the techniques presented in this paper can be used to design an optimal beam monitoring system. The interesting point in these methods is that they allow the simultaneous derivation of transverse and longitudinal optical functions without varying any machine parameter.

Numerical simulations of the transfer line joining the PS and the SPS, have been carried out to validate the new techniques and to compare the accuracy of the new methods with that of the standard approach based on three monitors. The Twiss parameters derived with the standard approach are in very good agreement with those obtained by using the five-monitor and six-monitor methods. As far as the dispersion function and its derivative are concerned, the fivemonitor method allows to derive values in good agreement with the nominal ones. Furthermore, it proved to be quite robust as it allowed to derive $D, D^{\prime}$ even when $\delta$ is rather small and the contribution to the betatronic beam size of the dispersion is very small.

The six-monitor method gives results in very good agreement with the standard and the 
five-monitor methods. However, it is more sensible to the actual value of $\delta$ than the five-monitor method as shown by the results of the numerical simulations.

\section{Acknowledgements}

The authors would like to thank P. Bryant, D. Möhl, C. Serre and D. Warner for reading the manuscript and for their suggestions for improving this paper. M. G. and M. M. would like to thank P. Bryant for interesting discussions and improvements of this paper.

\section{References}

[1] The LHC Study Group, P. Lefèvre and T. Pettersson Eds., "The Large Hadron Collider: Conceptual Design Study", CERN AC (LHC) 95-05 (1995).

[2] F. Blas, R. Cappi, V. Chohan, D. Cornuet, G. Daems, D. Dekkers, R. Garoby, D. Grier, J. Gruber, E. Jensen, H. Koziol, A. Krusche, K. D. Metzmacher, F. Pedersen, J. Pedersen, U. Raich, J-P. Riunaud, J-P. Royer, M. Sassowsky, K. Schindl, H. Schönhauer, M. Thivent, H. Ullrich, F. Völker, "Conversion of the PS Complex as LHC Pre-Injector", CERN PS (DI) 97-48 (1997).

[3] P. Collier, B. Goddard, R. Jung, K. H. Kissler, T. Linnecar, F. Ruggiero, W. Scandale, K. Schindl, G. Schröder, E. Shaposhnikova, L. Vos, "The SPS as Injector for LHC: Conceptual Design", CERN SL (DI) 97-007 (1997).

[4] M. Lindroos ed., "Workshop on Automated Beam Steering and Shaping (ABS)", CERN 99-07 (1999).

[5] G. Arduini, M. Giovannozzi, K. Hanke, D. Manglunki, M. Martini, G. Métral, "Measurement and Optimisation of the PS-SPS Transfer Line Optics", CERN PS (CA) 99-022 (1999).

[6] F. Zimmermann, in Joint US-CERN-Japan-Russia School on Particle Accelerators: Beam Measurement, edited by S. I. Kurokawa et al. (World Sci., Singapore, 1999) p. 21-107.

[7] H. Wiedemann, "Particle accelerator physics, 1 : basic principles and linear beam dynamics", (Springer, Berlin, 1993).

[8] P. J. Bryant, K. Johnsen, "Circular Accelerators and Storage Rings", (Cambridge University Press, NY, 1993).

[9] M. Arruat, M. Martini, "The new standard method to measure emittance in the PS transfer lines", CERN PS (PA) 92-59 (1992).

[10] G. Arduini, M. Giovannozzi, K. Hanke, D. Manglunki, M. Martini, "Measurement of the Optical Parameters of a Transfer Line Using Multi-Profile Analysis", CERN PS (CA) 9832 (1998).

[11] J. Camas, G. Ferioli, J. J. Gras, R. Jung, "Screens versus SEM Grids for single pass measurements in SPS, LEP and LHC", CERN SL (BI) 95-62 (1995).

[12] R. Jung, "Emittance measurement and control for the SPS as Injector for LHC", CERN SL (DI) 99-007 (1999).

[13] H. Grote, F. C. Iselin, “The MAD Program, User's Reference Manual”, CERN SL (AP) 90-13 (1990). 


\section{A Derivation of optical and beam parameters in circular machines}

The five-monitor method derived in the text can be applied also to the case of a circular machine in which one single monitor is available: the beam width is then measured at the same azimuthal location by means of a non-destructive device. The transfer matrix between the first and the $i$ th monitor is here given by a suitable power of the one-turn matrix. By using the optical parameters of the circular machine, it is possible to write explicitly the one-turn map [8], namely

$$
\mathcal{T}=\left(\begin{array}{ccc}
C & S & \xi \\
C^{\prime} & S^{\prime} & \xi^{\prime} \\
0 & 0 & 1
\end{array}\right)
$$

where $C, S$ can be replaced with the well-known expressions

$$
\begin{array}{ll}
C=\cos 2 \pi Q+\alpha \sin 2 \pi Q & S=\beta \sin 2 \pi Q, \\
C^{\prime}=-\gamma \sin 2 \pi Q & S^{\prime}=\cos 2 \pi Q-\alpha \sin 2 \pi Q .
\end{array}
$$

Here $Q$ represents the tune of the machine and $\alpha, \beta, \gamma$ the nominal values of the Twiss parameters at the monitor location. The two quantities $\xi, \xi^{\prime}$ can be expressed in terms of $D, D^{\prime}$, the values of the dispersion function and its derivative at the monitor location, by imposing that

$$
\left(\begin{array}{c}
D \\
D^{\prime} \\
1
\end{array}\right)=\left(\begin{array}{ccc}
C & S & \xi \\
C^{\prime} & S^{\prime} & \xi^{\prime} \\
0 & 0 & 1
\end{array}\right)\left(\begin{array}{c}
D \\
D^{\prime} \\
1
\end{array}\right),
$$

which corresponds to the periodic property of $D$ and $D^{\prime}$. The solution can also be expressed in matrix form

$$
\left(\begin{array}{c}
\xi \\
\xi^{\prime}
\end{array}\right)=\left(I-\mathcal{T}_{2 \times 2}\right)\left(\begin{array}{c}
D \\
D^{\prime}
\end{array}\right),
$$

where $\mathcal{T}_{2 \times 2}$ is the $2 \times 2$ sub-matrix of $\mathcal{T}$ and $I$ is the identity matrix.

In a circular machine, the matrix $\mathcal{T}_{i}$, expressing the beam dynamics between the first and the $i$ th monitor, is simply given by $\mathcal{T}_{i}=\mathcal{T}^{i}$ (i.e. the transfer matrix over $i$ consecutive turns). Furthermore, it is possible to show that

$$
\mathcal{T}^{i}=\left(\begin{array}{ccc}
C_{i} & S_{i} & \xi_{i} \\
C_{i}^{\prime} & S_{i}^{\prime} & \xi_{i}^{\prime} \\
0 & 0 & 1
\end{array}\right)
$$

where

$$
\begin{array}{ll}
C=\cos 2 \pi i Q+\alpha \sin 2 \pi i Q & S=\beta \sin 2 \pi i Q, \\
C^{\prime}=-\gamma \sin 2 \pi i Q & S^{\prime}=\cos 2 \pi i Q-\alpha \sin 2 \pi i Q .
\end{array}
$$

and

$$
\left(\begin{array}{l}
\xi_{i} \\
\xi_{i}^{\prime}
\end{array}\right)=\left(I-\mathcal{T}_{2 \times 2}^{i}\right)\left(\begin{array}{c}
D \\
D^{\prime}
\end{array}\right) .
$$

The previous relations can be used to parametrise the matrix $\mathcal{M}$ in Eq. (16), used to compute the optical parameters, hence allowing to derive an explicit expression for the determinant 
of $\mathcal{M}$ as a function of the nominal optical parameters and the number of turns $n_{i}$ between measurement $i$ and $i+1$, namely

$$
\begin{aligned}
\operatorname{det} \mathcal{M}= & -512 D^{2} \beta^{4} \sin \pi n_{1} Q \sin \pi n_{2} Q \sin \pi n_{3} Q \sin \pi n_{4} Q \\
& \sin \pi\left(n_{1}+n_{2}\right) Q \sin \pi\left(n_{2}+n_{3}\right) Q \sin \pi\left(n_{3}+n_{4}\right) Q \\
& \sin \pi\left(n_{1}+n_{2}+n_{3}\right) Q \sin \pi\left(n_{2}+n_{3}+n_{4}\right) Q \sin \pi\left(n_{1}+n_{2}+n_{3}+n_{4}\right) Q .
\end{aligned}
$$

From Eq. (41) it is evident that in order for $\mathcal{M}$ to be non-singular, the value of the dispersion function $D$ must be different from zero and that the following relation must be fulfilled

$$
\left(n_{1}+n_{2}+n_{3}+n_{4}\right) Q \neq p \quad \forall n_{i} \in \mathbb{N}, n_{i}>0 \text { and } p \in \mathbb{Z}
$$

The free parameters $n_{i}$ can be used to optimise the accuracy of the method. 